\title{
MEPE loss-of-function variant associates with decreased bone mineral density and increased fracture risk
}

Ida Surakka1,15, Lars G. Fritsche (10 1,2,15, Wei Zhou (i) 3,4, Joshua Backman', Jack A. Kosmicki ${ }^{5}$, Haocheng Lu (i) ${ }^{1}$, Ben Brumpton 6,7,8, Jonas B. Nielsen (1) ${ }^{1}$, Maiken E. Gabrielsen ${ }^{6}$, Anne Heidi Skogholt ${ }^{6}$, Brooke Wolford (1) ${ }^{4}$, Sarah E. Graham (1) 1, Y. Eugene Chen', Seunggeun Lee (i) ${ }^{2}$, Hyun Min Kang ${ }^{2}$, Arnulf Langhammer ${ }^{9}$, Siri Forsmo 9 , Bjørn O. Åsvold (10 6,9,10, Unnur Styrkarsdottir (10 11, Hilma Holm (1) 11, Daniel Gudbjartsson (1) 11,12, Kari Stefansson ${ }^{11,13}$, Aris Baras $\mathbb{D}^{5}$, Regeneron Genetics Center ${ }^{5, *}$, Goncalo R. Abecasis ${ }^{2,5}$, Kristian Hveem ${ }^{6,9 凶} \&$ Cristen J. Willer (1) 1,4,6,14凶

A major challenge in genetic association studies is that most associated variants fall in the non-coding part of the human genome. We searched for variants associated with bone mineral density (BMD) after enriching the discovery cohort for loss-of-function (LoF) mutations by sequencing a subset of the Nord-Trøndelag Health Study, followed by imputation in the remaining sample $(N=19,705)$, and identified ten known BMD loci. However, one previously unreported variant, LoF mutation in MEPE, p.(Lys70llefsTer26, minor allele frequency $[\mathrm{MAF}]=0.8 \%)$, was associated with decreased ultradistal forearm BMD $\left(P\right.$-value $\left.=2.1 \times 10^{-18}\right)$, and increased osteoporosis $\left(P\right.$-value $\left.=4.2 \times 10^{-5}\right)$ and fracture risk $\left(P\right.$-value $\left.=1.6 \times 10^{-5}\right)$. The MEPE LoF association with BMD and fractures was further evaluated in 279,435 UK (MAF $=0.05 \%$, heel bone estimated $\mathrm{BMD} P$-value $=1.2 \times 10^{-16}$, any fracture $P$-value $=0.05)$ and 375,984 Icelandic samples $(M A F=0.03 \%$, arm $B M D$ $P$-value $=0.12$, forearm fracture $P$-value $=0.005)$. Screening for the MEPE LoF mutations before adulthood could potentially prevent osteoporosis and fractures due to the lifelong effect on BMD observed in the study. A key implication for precision medicine is that highimpact functional variants missing from the publicly available cosmopolitan panels could be clinically more relevant than polygenic risk scores.

\footnotetext{
${ }^{1}$ Division of Cardiovascular Medicine, Department of Internal Medicine, University of Michigan, 1500 E. Medical Center Dr., Ann Arbor, MI 48109, USA.

2 Department of Biostatistics and Center for Statistical Genetics, University of Michigan School of Public Health, 1415 Washington Heights, 1700 SPH I, Ann Arbor, MI 48109, USA. ${ }^{3}$ Program in Medical and Population Genetics, Broad Institute of Harvard and MIT, 415 Main Street, Cambridge, MA 02142, USA. ${ }^{4}$ Department of Computational Medicine and Bioinformatics, University of Michigan, Palmer Commons, 100 Washtenaw Avenue, Ann Arbor, MI 48109, USA. ${ }^{5}$ Regeneron Genetics Center, 777 Old Saw Mill River Road, Tarrytown, NY 10591, USA. ${ }^{6}$ K.G. Jebsen Center for Genetic Epidemiology, Department of Public Health and Nursing, NTNU, Norwegian University of Science and Technology, NO-7491 Trondheim, Norway. ${ }^{7}$ MRC Integrative Epidemiology Unit, University of Bristol, Oakfield House, Oakfield Grove, Bristol BS8 2BN, UK. ${ }^{8}$ Clinic of Thoracic and Occupational Medicine, St. Olavs Hospital, Trondheim University Hospital, Prinsesse Kristinas gate 3, 7030 Trondheim, Norway. ${ }^{9}$ HUNT Research Centre, Department of Public Health and Nursing, Norwegian University of Science and Technology, Postboks 8905, N-7491 Levanger, Norway. ${ }^{10}$ Department of Endocrinology, St. Olavs Hospital, Trondheim University Hospital, Prinsesse Kristinas gate 3,7030 Trondheim, Norway. ${ }^{11}$ deCODE genetics/Amgen, Inc., Sturlugata 8, 101 Reykjavik, Iceland. ${ }^{12}$ School of Engineering and Natural Sciences, University of Iceland, Sturlugata 7, 101 Reykjavik, Iceland. ${ }^{13}$ Faculty of Medicine, University of Iceland, Vatnsmýrarvegur 16, 101 Reykjavik, Iceland. ${ }^{14}$ Department of Human Genetics, University of Michigan, 4909 Buhl Building, 1241 E. Catherine St, Ann Arbor, MI 48109, USA. ${ }^{15}$ These authors contributed equally: Ida Surakka, Lars G. Fritsche.

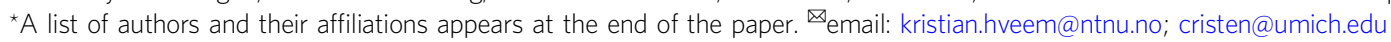


T he mineral content of bone reaches peak during young adulthood; as humans age, the mineral content of bone decreases and porosity increases, weakening the bones and leaving them vulnerable to fracture. Measurements of the density of bones, typically determined by $\mathrm{x}$-ray absorption, can predict which individuals are at risk of hip, vertebral and other fractures but are often performed clinically only after a fracture occurs ${ }^{1}$. Bisphosphonate and other oral, subcutaneous and intravenous medications can be prescribed to increase bone mineral density (BMD) and reduce fracture risk in osteoporotic individuals ${ }^{2}$. BMD is typically measured in individuals with high risk of osteoporosis (for example, due to family history of osteoporosis, use of corticosteroids or use of antiestrogen in breast cancer treatment) and is recommended to be tested after a fracture.

BMD is a complex trait with a strong genetic component; heritability estimates range between 50 and $85 \% \%^{3-6}$ and genomewide association studies demonstrate this is mostly through polygenic effects. Additionally, there are multiple forms of monogenic skeletal diseases caused by single mutations ${ }^{7}$, but these variants are typically very rare $(<1 / 1000)$. Genome-wide studies of estimated BMD, as measured by ultrasound of heel have identified almost 900 associated genomic regions ${ }^{8-10}$, with a substantial number also associated with fracture. Genomic discovery can aid in identifying targets for novel therapeutics, and potentially for identification of individuals at-risk for fracture that may benefit from preventive therapies.

Human diseases have typically been studied by testing associations between human genetic variation and phenotypes, where the discovered variants and genes are often investigated experimentally in model organisms or cell-based systems that can be genome-edited or perturbed in the laboratory. On the one hand, studying genetic mutations in humans themselves provides the natural genetic background and environmental conditions that lead to disease, but we are limited to observing the genetic changes that have arisen spontaneously in the human genome over time, and the frequency spectrum of variants that can be tested is limited by technology, cost and presence of those variants in the population under study. While on the other hand, the study of animal models can often provide conflicting or uninterpretable results when applied to humans, sometimes resulting in expensive clinical trials that fail.

To advance the translation of genetic discovery to improved therapeutics and prevention via prediction of at-risk individuals, we sought to identify rare and low-frequency loss-of-function $(\mathrm{LoF})$ variants associated with BMD and fractures through a genome-wide association study (GWAS). We employed methodology wherein we performed low-pass sequencing of a subset of the sample $(N=2202)$, then imputed variants, including insertion/deletion polymorphisms, into the remainder of the HUNT discovery sample $(N=19,705)$ followed by replication of previously unreported variants in two independent replication samples: UK Biobank $(N=279,435)$ and $\operatorname{deCODE}(N=$ 170,000). Using this approach, we identify a LoF mutation in $M E P E$, which may be useful for precision medicine and therapeutic development.

\section{Results}

Genome-wide screen for BMD-associated LoF variants. The Nord-Trøndelag Health Study (HUNT) ${ }^{11}$ performed screening of BMD during enrollment into the HUNT study at different time points: HUNT2 in 1995-1997 and HUNT3 in 2006-2008. The standard technology in use at that time was single-energy $\mathrm{x}$-ray absorptiometry (SXA), and the decision was made to focus on ultradistal forearm BMD measurements. Although this is not the current standard used in clinic or hospital-based cohort collections, the HUNT study has the advantage of a populationbased screening of individuals with a wide variety of ages, with the inclusion of healthy individuals relative to a clinic-based phenotype, and decades of longitudinal clinical follow-up including fractures. Furthermore, it has been demonstrated that the T-score derived from wrist BMD is correlated with hip Tscore $(r=0.61)$ and lumbar T-score $(r=0.53)^{12}$, suggesting that ultradistal forearm BMD is helpful for estimating risk of fractures as well as diagnosing osteoporosis ${ }^{13}$.

To enrich the discovery cohort for rare loss-of-function (LoF) variants typically missed by array-based genotyping, we first performed low-pass whole genome sequencing $(N=2202$, on average $5 \mathrm{X}$ coverage) followed by imputation into the remaining HUNT samples, and tested for association with $11.2 \mathrm{M}$ single nucleotide variants and 430,000 indels with high imputation quality (imputation $R^{2}>0.9$ ) and minor allele count $>10$ in 19,705 samples. We replicated 10 previously identified BMD loci with genome-wide significant associations with ultradistal forearm BMD (association test $P$-value $<5 \times 10^{-8}$, Fig. 1 , Table 1 ). One of the BMD-associated loci, MEPE on chromosome 4, spanned over a $5 \mathrm{Mb}$ window and contained the lead intergenic variant reported for association with femoral neck and lumbar spine BMD by the GEFOS consortium in 2009 (rs1471403 ${ }^{14}$ ) as well as the lead variant from the UK Biobank estimated heel bone mineral density (eBMD) GWAS ${ }^{10}$ (rs11934731; $r^{2}=0.71$ with GEFOS lead variant). In the HUNT discovery cohort $(N=19,705)$, the minor allele at the lead single nucleotide variant at this locus, rs181831514, had a much higher impact (effect $=-0.53 \mathrm{SD}$ [standard deviation units], minor allele frequency [MAF] $=$ $0.8 \%$ ), was much less common than the previously identified lead variants and was in nearly perfect linkage disequilibrium $\left(r^{2}=\right.$ 0.999) with a rare LoF indel in the MEPE gene (rs753138805, $M E P E$ p.Lys70IlefsTer26, $P$-value $=2.1 \times 10^{-18}$ ). The insertion/ deletion polymorphism was only observed following imputation from HUNT low-pass sequences which included indel calling. The Haplotype Reference Consortium imputation panel (which contains 1254 HUNT low-pass sequences which we submitted) was able to impute the intronic proxy variant (rs181831514; imputation $R^{2}=0.99$ ) but the indel was not present. The 1000 Genomes reference panel, which does include indel calls does not have p.Lys70IlefsTer26 present; however, the proxy variant imputation quality (imputation $R^{2}$ ) from 1000 Genomes reference dataset was 0.9979 .

Statistical evidence for MEPE p.Lys70IlefsTer26. MEPE p.Lys70IlefsTer 26 is located only $65 \mathrm{~kb}$ away from the previously identified association lead variant from a previous GWAS for eBMD in white British individuals ${ }^{8}$ (LD $r^{2}=0.06$ ). The nominally significant association signal at this lead variant (rs11934731, effect size for the minor allele $=0.029 \mathrm{SD}, P$-value $=0.01, \mathrm{MAF}=33 \%$ ) was slightly attenuated when we performed conditional analysis with MEPE p.Lys70IlefsTer26 as a covariate in the model (effect size $_{\text {conditional }}=0.021 \mathrm{SD}, \quad P$-value conditional $_{\text {com }}=0.06 ;$ Supplementary Fig. 1, Supplementary Data 1). Additionally, the association for the $M E P E$ LoF variant remains highly significant when conditioning for the lead variant of the eBMD analysis (effect size $_{\text {conditional }}=$ -0.529 SD, $P$-value conditional $\left.=5.3 \times 10^{-18}\right)$.

The LoF deletion demonstrated a very strong association with ultradistal forearm BMD (effect size $=-0.53 \mathrm{SD}, \quad N=19,705$, $P$-value $=2.1 \times 10^{-18}$, Table 2 ) and has an eight fold stronger impact on $\mathrm{BMD}$ than the common variant previously reported as associated with femoral neck and lumbar spine BMD by the GEFOS Consortium ${ }^{14}$ at this locus (effect size $=-0.07 \mathrm{SD}$, $\mathrm{MAF}=0.34)$. We replicated this finding in exome sequence data from 279,435 UK Biobank individuals with estimated heel BMD 
a

$11,603,265$ variants

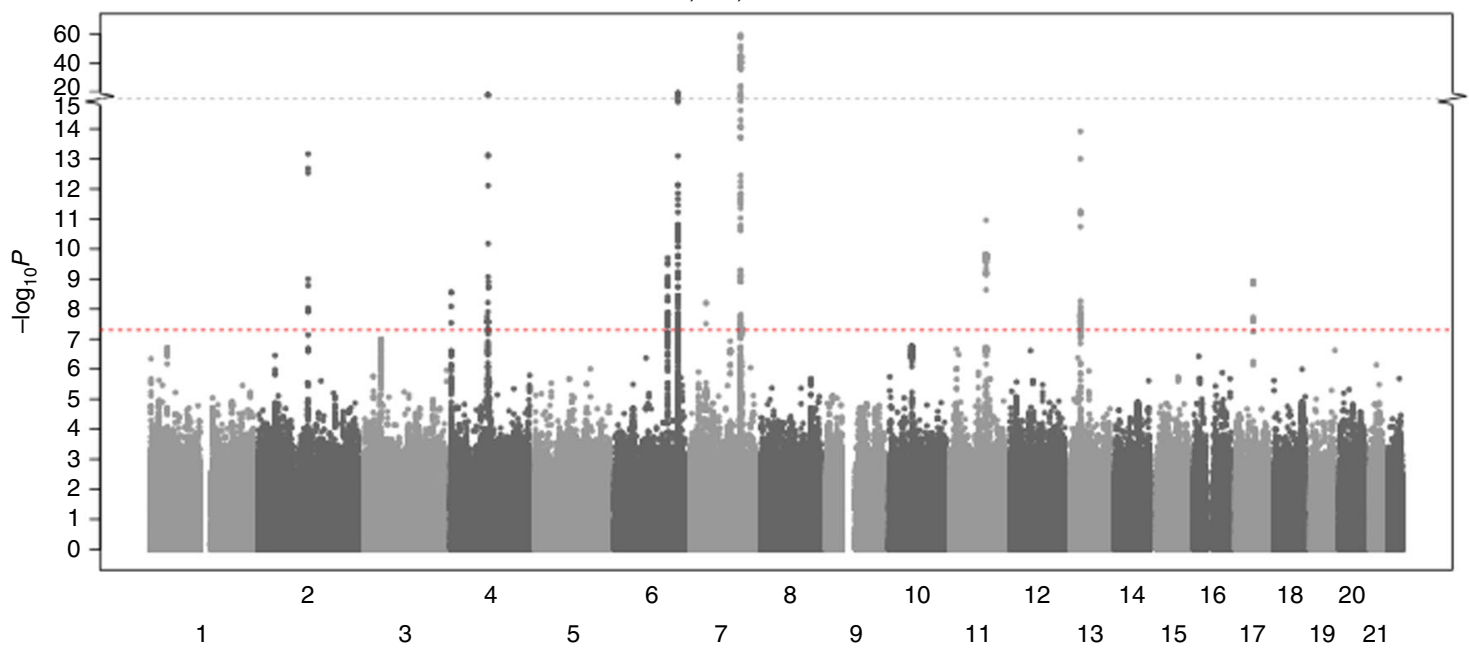

b

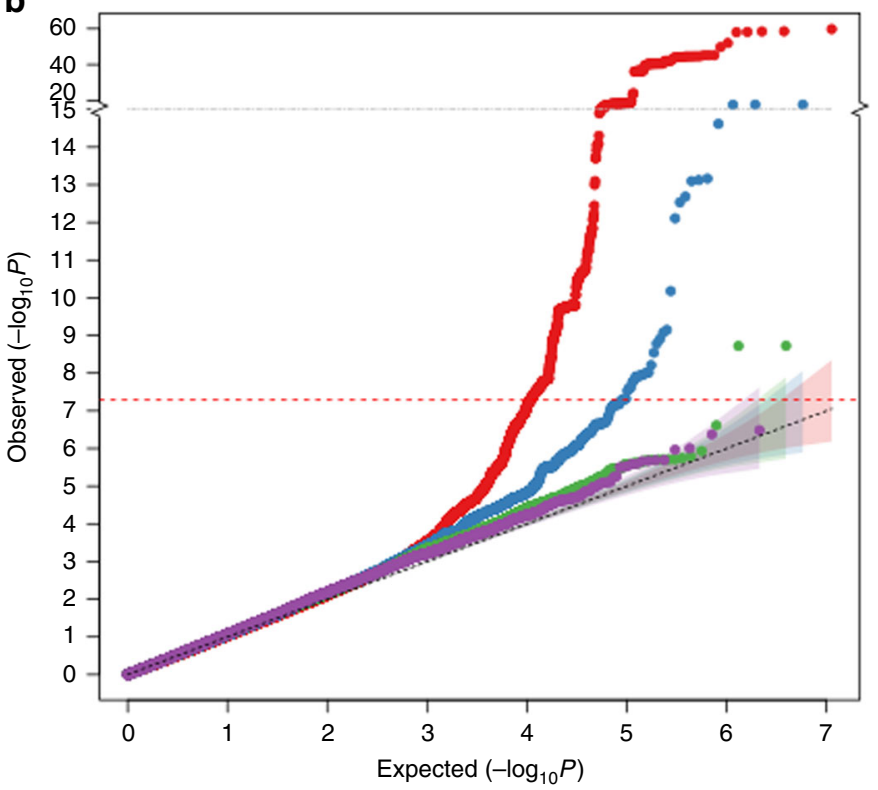

Fig. 1 Ultradistal forearm BMD Genome-wide association analysis results. Manhattan (upper panel) and QQ-plot (lower panel) in HUNT dataset ( $N=$ $19,705)$ for ultradistal forearm bone mineral density (BMD) genome-wide association analysis. In the Manhattan plot (upper panel) the genome-wide significance threshold $\left(P\right.$-value $<5 \times 10^{-8}$ ) is shown using a red dotted line. In the QQ-plot (lower panel), the tested variants have been divided into four groups based on MAF (red dots = MAF [0.05; 0.5], blue dots = MAF [0.005; 0.05], green dots = MAF [0.001; 0.005], purple dots = MAF [0.000253; 0.001]). MAF: minor allele frequency. $-\log _{10} P:-1 \times$ tenth logarithm of the association test $P$-value.

(eBMD), which demonstrated a genome-wide significant association with a similar effect size (effect size $=-0.48 \mathrm{SD}, N=279,435$, $P$-value $=1.2 \times 10^{-16}$, Table 2 ) as observed in Norwegian HUNT individuals. The proportion of UK individuals carrying at least one copy of MEPE p.Lys70IlefsTer26 was nearly 17 fold lower (0.095\%) than the frequency observed in Norwegian HUNT individuals (1.6\%). Additionally, this mutation shows comparable effect size (effect size for dual energy x-ray absorptiometry [DXA] arm $\mathrm{BMD}=-0.588, N=15,092)$ in the Icelandic population where, however, the variant is even more rare $(\mathrm{MAF}=0.03 \%)$, and thus lacks the power to replicate the association $(P$-value $=0.12)$.

MEPE LoF variant clinical characterization. To determine the impact of MEPE p.Lys70IlefsTer26 on clinical end points, we performed association analyses for bone-related phenotypes in HUNT (Supplementary Data 2) in the full HUNT dataset with genetic information available $(N=69,716)$. MEPE p.Lys70IlefsTer26 carriers show higher risk for multiple types of fractures as well as osteoporosis with odds ratios (OR) ranging between 1.35 and 2.06 (Table 3). We see similar ORs in the Icelandic replication dataset, but the only significant association is for the forearm fractures of old individuals (Supplementary Table 1). In the UK population we see nominally significant association to any fracture $(\mathrm{OR}=1.76$ [1.00; 3.11], Supplementary Table 1).

Typical diagnostic criteria for osteoporosis is a BMD more than $2.5 \mathrm{SD}$ below the reference population average ( $\mathrm{T}$-score $<$ $-2.5)$. As we did not have the official T-score available for the dataset, which is always in relation to a reference dataset BMD distribution, we used the ultradistal forearm BMD lower than $-2.5 \mathrm{SD}$ as a proxy. Within the HUNT participants with genotypes and BMD measured $(N=19,705)$, the proportion of individuals who experienced any fracture during an average of 31 
Table 1 Genome-wide significant loci in the discovery HUNT sample.

\begin{tabular}{|c|c|c|c|c|c|c|c|c|}
\hline rsID & Chromosome & $\begin{array}{l}\text { Position } \\
\text { (hg19) }\end{array}$ & $\begin{array}{l}\text { Effect allele/ } \\
\text { non-effect } \\
\text { allele }\end{array}$ & $\begin{array}{l}\text { Annotation } \\
\text { (candidate gene) }\end{array}$ & $\begin{array}{l}\text { Effect allele } \\
\text { frequency }\end{array}$ & $\begin{array}{l}\text { Imputation } \\
\text { quality }\left(R^{2}\right)\end{array}$ & $\begin{array}{l}\text { Effect } \\
\text { size }^{\mathrm{a}} \text { (SE) }\end{array}$ & $\begin{array}{l}\text { Association } \\
\text { test } P \text {-value }\end{array}$ \\
\hline rs115242848 & 2 & 119507607 & $\mathrm{~T} / \mathrm{C}$ & Intergenic (EN1) & 0.011 & 0.966 & $0.387(0.052)$ & $6.9 \times 10^{-14}$ \\
\hline rs181831514 & 4 & 88822746 & $\mathrm{~T} / \mathrm{C}$ & Intergenic (MEPE) & 0.008 & 0.988 & $-0.533(0.061)$ & $2.1 \times 10^{-18}$ \\
\hline rs7741021 & 6 & 127468274 & $\mathrm{C} / \mathrm{A}$ & Intronic (RSPO3) & 0.474 & 0.998 & $0.068(0.011)$ & $2.0 \times 10^{-10}$ \\
\hline rs4869742 & 6 & 151907748 & $\mathrm{~T} / \mathrm{C}$ & Intronic (CCDC170) & 0.273 & 0.992 & $-0.108(0.012)$ & $2.4 \times 10^{-19}$ \\
\hline rs489247 & 11 & 86881641 & $G / A$ & Intronic (TMEM135) & 0.258 & 0.997 & $-0.083(0.012)$ & $1.1 \times 10^{-11}$ \\
\hline rs2147161 & 13 & 42982302 & $\mathrm{C} / \mathrm{A}$ & Intergenic (TNSFS11) & 0.701 & 0.957 & $-0.091(0.012)$ & $1.2 \times 10^{-14}$ \\
\hline rs76410205 & 17 & 41807508 & $\mathrm{~T} / \mathrm{C}$ & $\begin{array}{l}\text { Intergenic } \\
\text { (SOST/DUSP3) }\end{array}$ & 0.096 & 0.971 & $0.111(0.018)$ & $1.2 \times 10^{-9}$ \\
\hline
\end{tabular}

This table shows the 10 genome-wide significant $\left(P\right.$-value $\left.<5 \times 10^{-8}\right)$ loci associated to ultradistal forearm bone mineral density $(B M D)$ in the discovery dataset $(N=19,705)$. As all these are previously known loci, the candidate gene has been taken from the previous publications. The effect of a variant is presented with the SAIGE linear mixed model effect size (Effect size) and standard error (SE) and the significance using the uncorrected two tailed Z-test $P$-value.

rsID reference SNP cluster ID, SE standard error of the effect estimate.

a Measured in SD units.

Table 2 Association of the MEPE LoF variant to BMD phenotypes in the three study datasets.

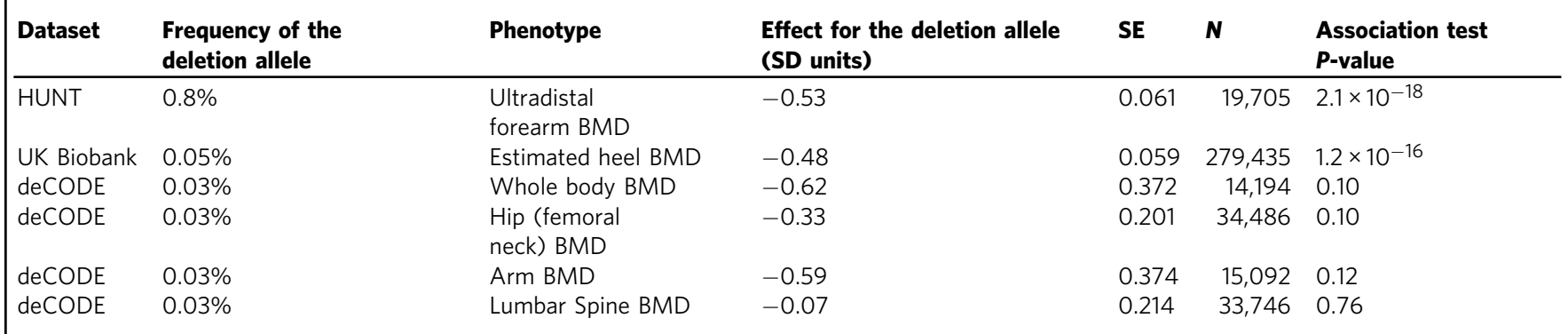

This table shows the association results for all three datasets (HUNT, UK Biobank and deCODE) and all tested bone mineral density phenotypes for the MEPE loss-of-function frameshift deletion, $\mathrm{p}$. Lys70llefsTer26 (rs753138805, chr4: 88766219 GAAA/-). The effect of a variant is presented with the effect size (Effect size) and standard error (SE) and the significance using the uncorrected two tailed Z-test $P$-value.

$B M D$ bone mineral density, SD standard deviation, SE standard error of the effect estimate, $N$ number of samples.

Table 3 Significant PheWAS results for the MEPE LoF mutation in HUNT dataset.

\begin{tabular}{lccc} 
Description & OR $[\mathbf{9 5 \%}$ CI] & Association test P-value & \#cases/\#controls \\
\hline Fracture of ankle and foot & $1.83[1.42 ; 2.35]$ & $3.3 \times 10^{-6}$ & $5478 / 45480$ \\
Fracture of upper limb & $1.51[1.26 ; 1.82]$ & $1.2 \times 10^{-5}$ & $11128 / 45480$ \\
Any fracture & $1.35[1.18 ; 1.54]$ & $1.6 \times 10^{-5}$ & $24155 / 45480$ \\
Fracture of radius and ulna & $1.61[1.29 ; 2.00]$ & $1.8 \times 10^{-5}$ & $7998 / 45480$ \\
Fracture of foot & $2.06[1.48 ; 2.86]$ & $2.0 \times 10^{-5}$ & $3223 / 45480$ \\
Osteoporosis & $1.58[1.27 ; 1.97]$ & $4.2 \times 10^{-5}$ & $6994 / 61558$ \\
Osteoporosis, osteopenia and pathological fracture & $1.50[1.22 ; 1.84]$ & $1.1 \times 10^{-4}$ & $8077 / 61558$ \\
Senile osteoporosis & $1.69[1.28 ; 2.22]$ & $1.8 \times 10^{-4}$ & $4482 / 61558$ \\
Fracture of humerus & $2.01[1.39 ; 2.90]$ & $2.0 \times 10^{-4}$ & $2457 / 45480$ \\
Fracture of unspecified bones & $1.46[1.19 ; 1.79]$ & $3.4 \times 10^{-4}$ & $8627 / 45480$ \\
Fracture of hand or wrist & $1.52[1.19 ; 1.94]$ & $7.7 \times 10^{-4}$ & $5860 / 45480$ \\
\hline This table presents all significant (P-value $<1.2 \times 10^{-3}$, Bonferroni correction for 42 phenotypes) end-point associations for the MEPE LoF frameshift deletion in HUNT dataset $(N=69,716)$. The effect of \\
a variant is presented with the odds ratio (OR) and $95 \%$ confidence intervals (CI) and the significance with uncorrected two tailed Z-test (for log(OR)) P-value. Full phenome-wide association scan \\
(PheWAS) results and ICD codes underlying the phenotypes can be found from Supplementary Data 2. \\
OR odds ratio, Cl confidence interval.
\end{tabular}

years of follow-up was $41.0 \%(N=8082)$ - this includes all types of fractures and all causes including trauma. Within the relatively small subset of individuals with ultradistal forearm BMD $<$ $-2.5 \mathrm{SD}$ at the time of BMD measurement, $53.3 \%$ had experienced a fracture (65 of 122; Supplementary Table 2). Simi- larly, almost half (49.0\%) of the individuals that carried the p.Lys70IlefsTer26 mutation had experienced a fracture (149 of 304 , OR $=1.39[1.11 ; 1.74])$ during the follow-up period, which was not significantly different from those with low BMD (BMD < $-2.5 \mathrm{SD}, \mathrm{OR}=1.65[1.15 ; 2.35])$. 


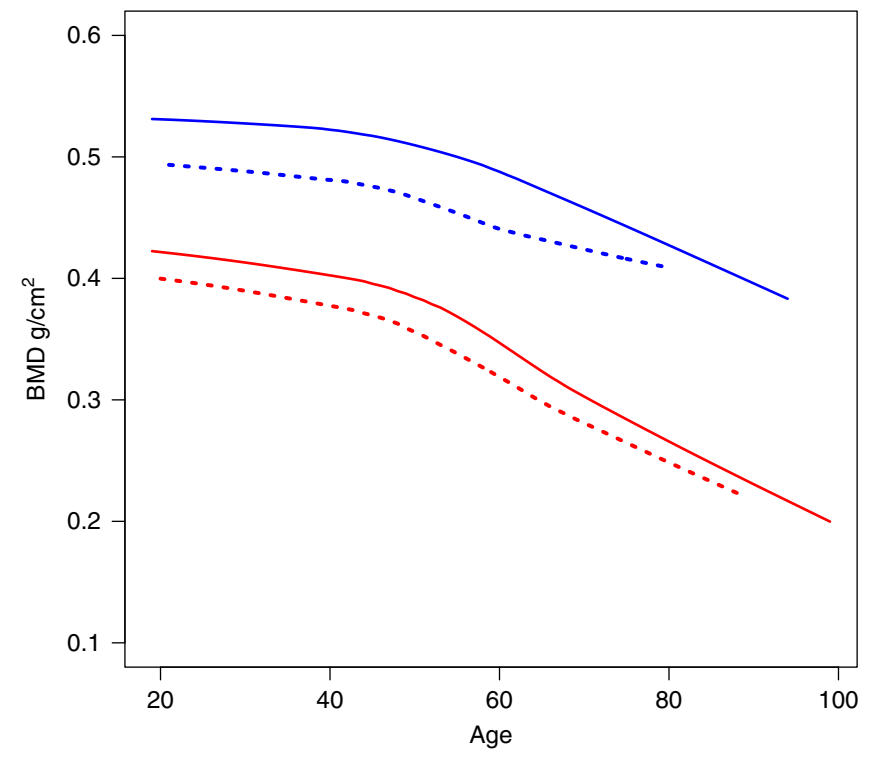

Fig. 2 Age trend in BMD for MEPE LoF mutation carriers. In this figure we have compared the forearm bone mineral density (BMD) in the MEPE lossof-function (LoF) mutation, p.Lys70llefsTer26, carriers (dotted lines)

compared to non-carriers (solid lines) in the HUNT dataset $(N=19,705)$. The trend over different ages is illustrated using LOWESS curve for males (blue lines) and females (red lines) separately.

To compare the potential clinical impact of this single variant, we calculated a polygenic score for eBMD in the HUNT dataset based on Kim et al. ${ }^{8}$ results using 1032 independently associated variants and weights from the UK Biobank cohort (Supplementary Data 3). Of the HUNT individuals in the lowest $1 \%$ of the BMD polygenic score distribution (i.e., $1 \%$ of the population with highest genetic burden for low BMD, fractures and osteoporosis), $38.2 \%$ experienced fracture during the follow-up, which is not significantly different from the rate in the remaining individuals $(\mathrm{OR}=0.89[0.66 ; 1.19]$, Fisher test $P$-value $=0.46$, Supplementary Table 2). We performed the same comparison for the $5 \%$ and $10 \%$ tails, and similarly saw no difference in the fracture rates $(\mathrm{OR}=0.98[0.86 ; 1.12]$, Fisher test $P$-value $=0.79$ and $1.04[0.94$; $1.14]$, Fisher test $P$-value $=0.44$, respectively).

When examining the decrease in ultradistal forearm BMD by age in both LoF carriers and non-carriers, we can see that the loss of BMD in both subgroups is the same (Fig. 2) suggesting that $M E P E$ p.Lys70IlefsTer26 mutation affects the BMD peak value, rather than the lifetime bone mass loss similarly to the LGR4 stop-gain variant previously identified by Styrkarsdottir et al. ${ }^{15}$. In our dataset, the ultradistal forearm BMD of an average 20 yearold woman carrying the LoF mutation in MEPE has a similar ultradistal forearm BMD as a 54 year-old non-carrier woman. A 20 year-old man carrying the LoF mutation in MEPE had an ultradistal forearm BMD similar to the average BMD for a 64 year-old male carrier (Supplementary Fig. 2).

\section{Discussion}

By sequencing followed by imputation into a large populationbased study in Norway, we identified a LoF mutation in the $M E P E$ gene, p.Lys70IlefsTer26, that demonstrates a genome-wide significant and high-impact association with ultradistal forearm $\mathrm{BMD}$ and with increased risk of osteoporosis and fractures. Common variants at this locus have been associated with BMDrelated traits in previous studies ${ }^{10,14}$, but we were able to pinpoint this association to a rare LoF mutation in the gene, definitively establishing a causal role and direction of effect of $M E P E$ on BMD.

Matrix extracellular phosphoglycoprotein (MEPE) was first cloned as a candidate for the oncogenic phosphaturic factor with similarities to a group of bone-tooth mineral matrix phosphoglycoproteins ${ }^{16}$. MEPE is expressed mainly in bone marrow and brain bone-associated tumors ${ }^{16}$. Mice with an ablated MEPE gene displayed increased osteoblast number, osteoblast activity and a higher bone mass ${ }^{17}$, whereas $M E P E$ overexpression in bone inhibited bone growth and mineralization in mice $^{18}$. Although how $M E P E$ regulates osteoblast activity remains unclear, $M E P E$ expression shows different patterns during osteoblast differentiation in human and mice. In mice, Mepe increases with differentiation ${ }^{19}$, while in human MEPE expression peaks in the proliferation phase and is suppressed during further differentiation ${ }^{20}$.

MEPE p.Lys70IlefsTer26 is the second rare LoF mutation found to be associated with BMD in population-based genomewide association studies. A study by Styrkarsdottir et al. ${ }^{15}$ found a rare stop-gain mutation in LGR4 gene with higher effect than the MEPE frameshift mutation (effect size for whole body $\mathrm{BMD}=$ $-0.75 \mathrm{SD}, P$-value $\left.=1.6 \times 10^{-6}, N=7359\right)$ that was associated with a binary phenotype for low hip, spine or whole body BMD (defined as $<-1.0 \mathrm{SD}, P$-value $\left.=1.3 \times 10^{-10}\right)$. The same research group has also identified two rare missense mutations in COL1A2 associated with low $\mathrm{BMD}^{21}$ in an Icelandic dataset with $N=$ 209,379 (p.Gly496Ala; MAF $=0.1 \%$, effect size for low spine $\mathrm{BMD}<-1 \mathrm{SD}=1.53, \quad P$-value $=1.8 \times 10^{-7}$ and p.Gly703Ser; $\mathrm{MAF}=0.05 \%$, effect size for low hip $\mathrm{BMD}<-1 \mathrm{SD}=2.23$, $P$-value $\left.=1.9 \times 10^{-8}\right)$. A study by Zheng et al. ${ }^{22}$ identified noncoding mutations with MAF 1\% in EN1 and WNT16 genes using whole-genome sequence data from the UK10K cohort $(N=$ 2882), followed by imputation into over 20,000 samples. Their results demonstrated a 4 -fold increase in the effect size for the low-frequency variants compared to the common variants found in the previous GWAS.

Because the mutation status can be determined at birth, $M E P E$ LoF carriers may benefit from treatment to preserve or increase peak BMD prior to the age of peak BMD. As the carriers show a similar rate of BMD loss during the adulthood as non-carriers, it may be sufficient for these individuals to be treated during the late childhood and early adulthood to increase the peak BMD. The promise of possible prevention of fractures by screening the population for MEPE mutations relative to measuring BMD is that MEPE p.Lys70IlefsTer26 carriers can be identified and provided treatment prior to decrease of BMD, which has the potential to prevent fractures in this subgroup and perhaps maintain higher lifetime BMD. Also, MEPE LoF carrier status identifies twice as many at-risk individuals as the $\mathrm{BMD}<-2.5 \mathrm{SD}$ criterion in HUNT study participants (1.54\% carry LoF vs $0.62 \%$ have $\mathrm{BMD}<-2.5 \mathrm{SD}$ ). We suggest that current clinical practice could be augmented with additional screening for the carriers of LoF variants of the MEPE gene. The finding that carriers of the mutation had lower ultradistal forearm BMD even during young adulthood, when bone mass would be expected to peak, suggests that these individuals may benefit from early initiation of osteoporosis prevention.

There are some limitations to our study. We acknowledge the difference between the discovery dataset and replication phenotypes, ultradistal forearm BMD measured with SXA (HUNT), compared to DXA from lumbar spine, hip, arm and whole body in deCODE and heel bone BMD measured with ultrasound in the UK biobank. However, as can be seen from Supplementary Fig. 3, the correlation between effect estimates for these two phenotypes is fairly high $(0.7-0.8)$ when comparing SNPs with adequate power in the smaller dataset. Additionally, as we have hospital registry data from the Nord-Trøndelag county only, it is possible 
that some of the HUNT study participants have experienced a fracture that is not accounted for in our dataset.

Mendelian randomization studies have demonstrated that $\mathrm{BMD}$ is a causal risk factor for fracture ${ }^{23}$. Therefore, we suggest that screening for individuals at high genetic risk could aid in starting appropriate pharmaceutical therapies and avoid fracture risk in these individuals. We performed low-pass whole genome sequencing of 2202 individuals followed by imputation into $\sim 20,000$ individuals from the HUNT study of Norway. We did not deeply sequence the MEPE gene in all Norwegians in this study, suggesting that additional LoF variants in this gene may be observed. By identifying other MEPE LoF mutations carriers, on top of the $1.6 \%$ with the p.Lys70IlefsTer26, we could increase the number of individuals who could be protected from fracture caused by low BMD. In addition to the European population, the MEPE LoF variant is present in African and Latino populations but with an extremely low allele frequency. However, given the presence of 16 different LoF mutations in the UK population $^{24}$, different LoF mutations may be present, but as-yetundetected in other populations.

This study demonstrates that continued investigation of genetic variation in humans, particularly rare variants identified through sequencing, can identify genetic variants that clearly and immediately define functional genes and may be useful for precision medicine and therapeutic development.

\begin{abstract}
Methods
HUNT genotype dataset. The discovery dataset, The Nord-Trøndelag Health Study (HUNT) ${ }^{11}$, is a population-based cohort of $\sim 120,000$ (descriptives in Supplementary Table 3) from the county of Nord-Trøndelag, Norway. Since 1984, phenotype data has been collected for these individuals approximately every 11 years. Participation in the HUNT Study is based on informed consent and the study has been approved by the Data Inspectorate and the Regional Ethics Committee for Medical Research in Norway (REK: 2014/144). In total, approximately 70,000 HUNT individuals have been genotyped using the Illumina Human CoreExome v1.1 array from both HUNT2 (collected between 1995-1997) and HUNT3 (collected between 2006 and 2008). After quality control of the genotype data, 69,716 European ancestry samples were imputed using a combined Haplotype Reference Consortium reference panel and a population specific whole genome sequence-based imputation panel ${ }^{25}$. 11.2 M single nucleotide variants and 430,000 indels with high imputation quality (imputation $R^{2}>0.9$ ) and minor allele count $>10$ were included in the analysis. Variants were annotated as a LoF mutation (3510 variants) if predicted as LoF (stop gain, stop loss, splice variant or frameshift) for either UCSC, Ensembl or RefSeq transcripts by ANNOVAR in Whole Genome Sequence Annotator ${ }^{26}$ v0.7.
\end{abstract}

HUNT phenotypes. BMD (in $\mathrm{g} / \mathrm{cm}^{2}$ ) was measured at the ultradistal part of the non-dominant forearm by single-energy x-ray absorptiometry (DTX100; Osteometer MediTech, Inc, Hawthorne, CA), and the measurements were standardized using equipment-specific correction factor ${ }^{27}$ estimated by three repeated hydroxyapatite bone imitation measurements of the European Forearm Phantom (QRM GmbH, Moehrendorf, Germany). BMD was measured in a subset of adult HUNT participants, including: $5 \%$ random samples of all participants, random samples of female participants in selected municipalities and age-groups within 35-85 years of age, and participants reporting ever having asthma, asthma-related symptoms or use of asthma medication (detailed selection criteria are available at ntnu.edu/hunt/databank). The present analyses were restricted to participants of European ancestry. For individuals who had their BMD measured in either HUNT2 collection, HUNT2 follow-up (2001) or HUNT3 collection, the HUNT2 measurement was prioritized, follow by HUNT3, then HUNT2 follow-up. The final discovery analysis dataset consisted of 19,705 samples with both imputed genome information and BMD.

Using the unique 11-digit national identification number that is allocated to all Norwegian citizens, we linked the HUNT study data to prospectively recorded information on fractures at the hospitals serving Nord-Trøndelag county: the local Levanger and Namsos Hospitals (Nord-Trøndelag Hospital Trust) and St. Olavs Hospital, Trondheim University Hospital. ICD-9 and ICD-10 codes from the electronic patient administrative systems were available from all hospitals from September, 1987 through October, 2017. For forearm and hip fractures at Levanger and Namsos Hospitals from October, 1995 through December, 2012, all diagnoses were validated by examination of medical records (relevant ICD codes accompanied by a procedure code for reduction, surgical intervention, or intervention with a rigid device), confirmation by X-ray or by review by a medical doctor ${ }^{28}$. A full list of PheCodes (derived from ICD-9 and ICD-10 codes) included in the phenome-wide association analysis can be found in Supplementary Data 2.

Statistical methods for discovery in HUNT. The association analysis in the discovery dataset was performed using SAIGE ${ }^{29}$, which implements linear or logistic mixed effects model (for quantitative and binary phenotypes respectively) accounting for sample relatedness and subtle population structure. The association analyses for inverse normal transformed ultradistal forearm BMD and clinical end points were adjusted with age (birth year for the clinical end points), sex, the first 4 genetic principal components and genotyping batch. Formal conditional analysis for the MEPE locus was performed using the same software, model and covariates as the discovery association analysis by adding the LoF variant as an additional covariate in the linear mixed model. Due to power restrictions, the analyses for clinical end points were restricted to PheCode-derived diagnoses with at least 500 cases. The sample size for the ultradistal forearm BMD association analysis was $N=19,705$ and for the clinical end-point analyses $N=69,716$. Clinical end points reaching $P$-value $<1.2 \times 10^{-3}$ (Bonferroni correction for 42 end points) were regarded as statistically significant. The LOWESS (Locally Weighted Scatterplot Smoothing) curve for age trend was fitted using smoother span (the proportion of points in the figure affecting the local value) of 2/3. The Fisher tests for comparing different predictors for fractures and all the Figures have been done using $\mathrm{R}$ (https://cran.r-project.org) v3.5.3.

Replication datasets. Replication of the association at the MEPE LoF variant, $\mathrm{p}$. Lys70IlefsTer26, was tested within the UK Biobank whole-exome sequence dataset in 279,435 participants. All participants in the UK Biobank provided informed consent and the study has obtained Research Tissue Bank (RTB) approval from its ethics committee (The Research Ethics Committee approval number; 11/NW/ 0382). Detailed cohort descriptions, sequencing, imputation and analysis methods for the UK biobank replication dataset can be found from Van Hout et al. ${ }^{24}$. Briefly, 302,342 participants (of which 279,435 with eBMD) were exome sequenced (coverage exceeds $20 \mathrm{X}$ at $95.5 \%$ of sites on average) resulting in $\sim 12$ million variants in targeted regions. Heel bone quality was evaluated with two methods; quantitative ultrasound speed of sound and broadband ultrasound attenuation using a Sahara Clinical Bone Sonometer (Hologic Corporation, Bedford, Massachusetts, USA $)^{10}$. Raw values for eBMD were first stratified by sex, rank-inverse normal transformed, and then re-combined. Association analysis was performed using a linear mixed model implemented in BOLT-LMM v2.3.2 (https://data. broadinstitute.org/alkesgroup/BOLT-LMM/) with covariates for age, age-squared, and first ten genetic principal components. The replication analysis for fractures (ICD codes S22-S92, excluding skull [S02] and neck [S12]) was ran using SAIGE with age, age ${ }^{2}$, age-sex interaction, sex and first ten genetic principal components as covariates.

The Icelandic replication dataset (deCODE) ${ }^{30,31}$ is based on 170,000 genotyped samples which have been imputed using a whole-genome sequenced population specific imputation panel. All participating individuals, or their guardians, gave their informed consent before blood samples were drawn and the study has been approved by the National Bioethics Committee and the Icelandic Data Protection Authority. Using these samples, the genotypes of 375,984 samples have been imputed using familial imputation. The imputation quality for the $M E P E$ p.Lys70IlefsTer26 (imputation info score) was 0.99. The BMD in the dataset has been measured using DXA from lumbar spine, hip, arm and whole body. Additionally, the dataset has health-care registry data available, which have been used in the end-point association replication.

\section{Data availability}

The GWAS summary statistics are available at http://csg.sph.umich.edu/willer/public/ bmd2020/. All other data that support the findings of this study are available from the corresponding author upon reasonable request. The Haplotype Reference Consortium imputation panel is accessible through the Michigan Imputation Server (https:// imputationserver.sph.umich.edu/index.html\#!). The UK Biobank replication cohort is a publicly available dataset for research purposes and can be accessed/applied from https:// www.ukbiobank.ac.uk. The deCODE Genetics dataset summary results can be requested from the deCODE authors.

Received: 6 December 2019; Accepted: 26 May 2020; Published online: 23 October 2020

\section{References}

1. Cauley, J. A., Hochberg, M. C. \& Lui, Ly. et al. Long-term risk of incident vertebral fractures. JAMA 298, 2761-2767 (2007).

2. Lorentzon, M. Treating osteoporosis to prevent fractures: current concepts and future developments. J. Intern. Med. 285, 381-394 (2019).

3. Howard, G. M., Nguyen, T. V., Harris, M., Kelly, P. J. \& Eisman, J. A. Genetic and environmental contributions to the association between quantitative 
ultrasound and bone mineral density measurements: a twin study. J. Bone Miner. Res. 13, 1318-1327 (1998).

4. Karasik, D., Myers, R. H. \& Hannan, M. T. et al. Mapping of quantitative ultrasound of the calcaneus bone to chromosome 1 by genome-wide linkage analysis. Osteoporos Int. 13, 796-802 (2002).

5. Hunter, D. J., de Lange, M., Andrew, T., Snieder, H., MacGregor, A. J. \& Spector, T. D. Genetic variation in bone mineral density and calcaneal ultrasound: a study of the influence of menopause using female twins. Osteoporos Int. 12, 406-411 (2001).

6. Arden, N. K., Baker, J., Hogg, C., Baan, K. \& Spector, T. D. The heritability of bone mineral density, ultrasound of the calcaneus and hip axis length: a study of postmenopausal twins. J. Bone Miner. Res. 11, 530-534 (1996).

7. Bonafe, L., Cormier-Daire, V. \& Hall, C. et al. Nosology and classification of genetic skeletal disorders: 2015 revision. Am. J. Med. Genet. A 167A, 2869-2892 (2015).

8. Kim, S. K. Identification of 613 new loci associated with heel bone mineral density and a polygenic risk score for bone mineral density, osteoporosis and fracture. PLOS ONE 13, e0200785 (2018).

9. Kemp, J. P., Morris, J. A. \& Medina-Gomez, C. et al. Identification of 153 new loci associated with heel bone mineral density and functional involvement of GPC6 in osteoporosis. Nat. Genet. 49, 1468-1475 (2017).

10. Morris, J. A., Kemp, J. P. \& Youlten, S. E. et al. An atlas of genetic influences on osteoporosis in humans and mice. Nat. Genet. 51, 258-266 (2019).

11. Krokstad, S., Langhammer, A. \& Hveem, K. et al. Cohort profile: the HUNT Study, Norway. Int. J. Epidemiol. 42, 968-977 (2013).

12. Azami, A., Anari, H., Iranparvar, M., Azizi, A. \& Habibzadeh, A. Comparison of bone mineral densitometry at 2 sites versus 3 sites in patients suspicious for osteoporosis. Clin. Med. Insights Arthritis Muscoloskelet. Disord. 12, 1179544119849017 (2019).

13. Eftekhar-Sadat, B., Ghavami, M., Toopchizadeh, V. \& Ghahvechi Akbari, M. Wrist bone mineral density utility in diagnosing hip osteoporosis in postmenopausal women. Ther. Adv. Endocrinol. Metab. 7, 207-211 (2016).

14. Rivadeneira, F., Styrkársdottir, U. \& Estrada, K. et al. Twenty bone-mineraldensity loci identified by large-scale meta-analysis of genome-wide association studies. Nat. Genet. 41, 1199-1206 (2009).

15. Styrkarsdottir, U., Thorleifsson, G. \& Sulem, P. et al. Nonsense mutation in the LGR4 gene is associated with several human diseases and other traits Nature. 497, 517-520 (2013).

16. Rowe, P. S., de Zoysa, P. A. \& Dong, R. et al. MEPE, a new gene expressed in bone marrow and tumors causing osteomalacia. Genomics. 67, 54-68 (2000).

17. Gowen, L. C., Petersen, D. N. \& Mansolf, A. L. et al. Targeted disruption of the osteoblast/osteocyte factor 45 gene (OF45) results in increased bone formation and bone mass. J. Biol. Chem. 278, 1998-2007 (2003).

18. David, V., Martin, A., Hedge, A. M. \& Rowe, P. S. Matrix extracellular phosphoglycoprotein (MEPE) is a new bone renal hormone and vascularization modulator. Endocrinology 150, 4012-4023 (2009).

19. Argiro, L., Desbarats, M. \& Glorieux, F. H. Ecarot B. Mepe, the gene encoding a tumor-secreted protein in oncogenic hypophosphatemic osteomalacia, is expressed in bone. Genomics. 74, 342-351 (2001).

20. Siggelkow, H., Schmidt, E., Hennies, B. \& Hüfner, M. Evidence of downregulation of matrix extracellular phosphoglycoprotein during terminal differentiation in human osteoblasts. Bone 35, 570-576 (2004).

21. Styrkarsdottir, U., Thorleifsson, G. \& Eiriksdottir, B. et al. Two rare mutations in the COL1A2 gene associate with low bone mineral density and fractures in Iceland. J. Bone Miner. Res. 31, 173-179 (2016).

22. Zheng, H. F., Forgetta, V. \& Hsu, Y. H. et al. Whole-genome sequencing identifies EN1 as a determinant of bone density and fracture. Nature. 526, 112-117 (2015).

23. Trajanoska, K., Morris, J. A. \& Oei, L. et al. Assessment of the genetic and clinical determinants of fracture risk: genome wide association and mendelian randomisation study. BMJ. 362, k3225 (2018).

24. Van Hout C. V., Tachmazidou I., Backman J. D. et al. Whole exome sequencing and characterization of coding variation in 49,960 individuals in the UK Biobank. Preprint at https://www.biorxiv.org/content/10.1101/ 572347v1 (2019).

25. Zhou, W., Fritsche, L. G. \& Das, S. et al. Improving power of association tests using multiple sets of imputed genotypes from distributed reference panels. Genet. Epidemiol. 41, 744-755 (2017).

26. Liu, X., White, S. \& Peng, B. et al. WGSA: an annotation pipeline for human genome sequencing studies. J. Med. Genet. 53, 111-112 (2016).

27. Langhammer, A., Forsmo, S., Lilleeng, S., Johnsen, R. \& Bjermer, L. Effect of inhaled corticosteroids on forearm bone mineral density: the HUNT study, Norway. Respir Med. 101, 1744-1752 (2007).

28. Hoff, M., Skurtveit, S. \& Meyer, H. E. et al. Use of anti-osteoporotic drugs in central Norway after a forearm fracture. Arch. Osteoporos. 10, 235 (2015).
29. Zhou, W., Nielsen, J. B. \& Fritsche, L. G. et al. Efficiently controlling for casecontrol imbalance and sample relatedness in large-scale genetic association studies. Nat. Genet. 50, 1335-1341 (2018)

30. Gudbjartsson, D. F., Helgason, H. \& Gudjonsson, S. A. et al. Large-scale whole-genome sequencing of the Icelandic population. Nat. Genet. 47, 435-444 (2015).

31. Styrkarsdottir, U., Stefansson, O. A. \& Gunnarsdottir, K. et al. GWAS of bone size yields twelve loci that also affect height, BMD, osteoarthritis or fractures. Nat. Commun. 10, 2054 (2019).

\section{Acknowledgements}

The authors thank all HUNT, UK Biobank and deCODE genetics study participants for their contributions to research. The Nord-Trøndelag Health Study (The HUNT Study) is a collaboration between HUNT Research Centre (Faculty of Medicine and Health Sciences, NTNU, Norwegian University of Science and Technology), Nord-Trøndelag County Council, Central Norway Regional Health Authority, and the Norwegian Institute of Public Health. The K.G. Jebsen Center for Genetic Epidemiology is financed by Stiftelsen Kristian Gerhard Jebsen; Faculty of Medicine and Health Sciences, Norwegian University of Science and Technology (NTNU); and Central Norway Regional Health Authority. The replication in UK Biobank has been conducted using the data application number 26041. J.B.N. was supported by grants from the Danish Heart Foundation and the Lundbeck Foundation. Y.E.C was supported by NIH grants R01-HL068878 and R01HL137214. C.J.W. was supported by NIH grants R35-HL135824, R01-HL127564, R01HL117626-02-S1, and R01-HL130705. I.S. is supported by a Precision Health Scholars Award from the University of Michigan Medical School. Regeneron Genetics Center authors are listed in alphabetical order. Detailed author contributions for Regeneron Genetics Centre authors can be found from Supplementary Note.

\section{Author contributions}

I.S., L.G.F., K.H., and C.J.W. designed the study. I.S., L.G.F., W.Z., H.L., B.B., J.B.N., B.W., and S.E.G. analyzed the data. H.L. and Y.E.C. evaluated functional follow-up experiments. L.G.F., J.B.N., M.E.G., A.H.S., A.L., S.F., B.O.Å. contributed to the phenotype harmonizations. J.B., J.A.K., U.S., H.H., D.G., K.S., A.B., Regeneron Genetics Center and G.R.A. contributed to the replications. W.Z., B.B., S.L., H.M.K., D.G., and G.R.A. provided statistical expertise. J.B.N., Y.E.C., A.L., S.F., B.O.Å., U.S., H.H., and K.H. provided clinical expertise. I.S., L.G.F., K.H., and C.J.W. wrote the paper. All the authors read and revised the manuscript.

\section{Competing interests}

G.R.A., J.B. J.A.K., and A.B. work at Regeneron. U.T., H.H., D.G., and K.S. are employed at deCODE genetics/Amgen Inc. The spouse of C.J.W. works at Regeneron. I.S, L.G.F, W.Z., H.L., B.B., J.B.N., M.E.G., A.H.S., B.W., S.E.G., Y.E.C., S.L., H.M.K., A.L., S.F., B.O.Å., and K.H., declare no competing interests.

\section{Additional information}

Supplementary information is available for this paper at https://doi.org/10.1038/s41467020-17315-0.

Correspondence and requests for materials should be addressed to K.H. or C.J.W.

Peer review information Nature Communications thanks Dominic Furniss, David Karasik, and the other, anonymous reviewer(s) for their contribution to the peer review of this work. Peer review reports are available.

Reprints and permission information is available at http://www.nature.com/reprints

Publisher's note Springer Nature remains neutral with regard to jurisdictional claims in published maps and institutional affiliations.

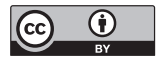

Open Access This article is licensed under a Creative Commons Attribution 4.0 International License, which permits use, sharing, adaptation, distribution and reproduction in any medium or format, as long as you give appropriate credit to the original author(s) and the source, provide a link to the Creative Commons license, and indicate if changes were made. The images or other third party material in this article are included in the article's Creative Commons license, unless indicated otherwise in a credit line to the material. If material is not included in the article's Creative Commons license and your intended use is not permitted by statutory regulation or exceeds the permitted use, you will need to obtain permission directly from the copyright holder. To view a copy of this license, visit http://creativecommons.org/ licenses/by/4.0\%.

(c) The Author(s) 2020 


\section{Regeneron Genetics Center}

Xiaodong Bai ${ }^{5}$, Suganthi Balasubramanian ${ }^{5}$, Leland Barnard ${ }^{5}$, Andrew Blumenfeld ${ }^{5}$, Michael Cantor ${ }^{5}$, Giovanni Coppola ${ }^{5}$, Aris Economides ${ }^{5}$, Gisu Eom ${ }^{5}$, Lukas Habegger ${ }^{5}$, Young Hahn ${ }^{5}$, Alicia Hawes ${ }^{5}$, Marcus B. Jones ${ }^{5}$, Shareef Khalid ${ }^{5}$, Luca A. Lotta ${ }^{5}$, Evan K. Maxwell ${ }^{5}$, Lyndon J. Mitnaul ${ }^{5}$, John D. Overton ${ }^{5}$, Jeffrey G. Reid ${ }^{5}$, Manuel Allen Revez Ferreira ${ }^{5}$, William Salerno ${ }^{5}$, Deepika Sharma ${ }^{5}$, Alan Shuldiner ${ }^{5}$, Jeffrey C. Staples ${ }^{5} \&$ Ashish Yadav 5

A full list of members appears in the Supplementary Information. 